\title{
STABILITY CONDITIONS FOR A NEUTRALISED ELECTRON COOLING BEAM
}

\author{
J. Bosser, S. Maury, I. Meshkov*, D. Möhl, E. Mustafin**, \\ E. Syresin*, F. Varenne, P. Zenkevich***
}

\begin{abstract}
Charge neutralisation of the cooling electron beam, e.g. by stationary ions produced from the residual gas, is desirable to compensate the space-charge induced velocity spread which tends to reduce the cooling force. However, it has been demonstrated by Parkhomchuk and collaborators that two-stream instabilities, especially transverse dipole modes, are a serious threat to the stability. In the present report we analyse stabilising mechanisms including Landau damping, external feedback and clearing of reflected electrons. Experimental results at LEAR (the Low Energy Antiproton Ring at CERN) are discussed in a companion paper at this conference.
\end{abstract}

*JINR, Dubna, Russia

* IHEP, Protvino, Russia

*** ITEP, Moscow, Russia

Paper presented at the 1995 Particle Accelerator Conference Dallas, USA, 30 April - 5 May 1995

Geneva, Switzerland

$10 / 5 / 95$ 


\title{
STABILITY CONDITIONS FOR A NEUTRALISED ELECTRON COOLING BEAM
}

\author{
J. Bosser, S. Maury, D. Möhl, and F. Varenne, CERN, CH-1211 Geneva 23 \\ I. Meshkov and E. Syresin, JINR, Dubna, Russia \\ E. Mustafin, IHEP, Protvino, Russia \\ P. Zenkevich, ITEP, Moscow, Russia
}

Charge neutralisation of the cooling electron beam, e.g. by stationary ions produced from the residual gas, is desirable to compensate the space-charge induced velocity spread which tends to reduce the cooling force. However, it has been demonstrated by Parkhomchuk and collaborators that two-stream instabilities, especially transverse dipole modes, are a serious threat to the stability. In the present report we analyse stabilising mechanisms including Landau damping, external feedback and clearing of reflected electrons. Experimental results at LEAR (the Low Energy Antiproton Ring at CERN) are discussed in a companion paper at this conference.

\section{INTRODUCTION}

It is known that the maximum current in a neutralised electron cooling system is constrained by transverse instabilities [1,2]. In previous papers Nezlin's theory for completely neutralised beams [3] was used. However, experiments at LEAR [4] with a vacuum of $10^{-11}$ torr show that the neutralisation is less than unity and therefore damping of the ion oscillations connected with reflections from the Debye boundary is absent. In such a system it is necessary to take into account the classical Landau damping due to the velocity spread of the neutralising ions.

We derive a dispersion relation to include this spread and discuss resulting stability conditions. Moreover, the influence of external forces on the beam motion is examined.

\section{EQUATIONS OF MOTION}

Let us consider a system including only the cooling electrons and the slow (neutralising) ions. For simplicity, we assume that the beams have uniform transverse densities. Then the equations of motion are :

$$
\begin{aligned}
& \frac{d^{2} \tilde{U}_{e}}{d t^{2}}+i \omega_{L e} \frac{d \tilde{U}_{e}}{d t}-\omega_{e l}^{2}\left(\tilde{U}_{e}-U_{e}\right)+ \\
& +\eta \gamma^{2} \omega_{e l}^{2}\left(\tilde{U}_{e}-U_{i}\right)=F_{e}(z) e^{-i \omega t} \\
& \frac{d^{2} \tilde{U}_{i}}{d t^{2}}+i \omega_{L i} \frac{d \tilde{U}_{i}}{d t}-\eta \omega_{i o n}^{2}\left(\tilde{U}_{i}-U_{i}\right)+ \\
& +\omega_{i o n}^{2}\left(\tilde{U}_{i}-U_{e}\right)=F_{i}(z) e^{-i \omega t}
\end{aligned}
$$

Here the subscripts $e$ and $i$ refer to electrons and ions respectively, $U=x+i y$ describes the horizontal $(x)$ and vertical $(y)$ displacement in complex notation, $\tilde{U}$ denotes the motion of a single ion and $U$ the motion of the beam centre of charge: $U=(1 / N) \Sigma \tilde{U}$, or if the dispersion is mainly due to the velocity spread $U=\int \tilde{U} f(v)$ dv where $f(v)$ is the velocity distribution normalized to $f(v) d v=1$. The derivatives in Eq. (1) have to be taken along the particle orbit: $d / d t=(\partial / \partial t)+v_{z}(\partial / \partial z)$, etc. The different frequencies have the following significance:

$\omega_{L e}=e B / m_{e}$ is the Larmor frequency (= cyclotron frequency) of the electrons in the longitudinal magnetic field of the cooler, $m_{e} \gamma$ is the relativistic mass of the electrons $\left(\omega_{L e}=2 \pi \times 1.75 \mathrm{GHz}\right)$,

$\omega_{L i} \quad=Z e B / M$ is the Larmor frequency of the ions with charge number $Z$ and mass $M=A m_{p}$ $\left(\omega_{L i}=2 \pi \times 957 \mathrm{kHz}\right)$,

$\omega_{e l}^{2} \quad=\left(2 \pi n_{e} / \gamma^{3}\right) r_{e} c^{2}$ describes the electron space-charge effect on an electron, $n_{e}$ is the electron density (electrons $/ \mathrm{m}^{3}$ ), $r_{e}$ is the classical electron radius, $\mathrm{c}$ is the velocity of light $\left(\omega_{e l}=2 \pi \times 35 \mathrm{MHz}\right)$,

$\omega_{i o n}^{2}=\left(2 \pi n_{e} Z / A\right) r_{p} c^{2}$ with $\omega_{i o n}$ the ion frequency in the space-charge potential of the electrons, $r_{p}$ is the classical proton radius $\left(\omega_{\text {ion }}=2 \pi \times 835 \mathrm{kHz}\right)$.

In the following we will use three more frequencies which we define here for convenience :

$\omega_{e}^{2}=\left(\eta \gamma^{2}-1\right) \omega_{e l}^{2}$ describes the incoherent space-charge effect experienced by the electrons, $\eta=Z n_{i} / n_{e}$ is the neutralisation factor with $n_{i}$ the density of the trapped ions $\left(\omega_{e}=2 \pi \times 27.4 \mathrm{MHz}\right)$,

$\omega_{i}^{2}=(1-\eta) \omega_{i o n}^{2}$ describes the incoherent space-charge effect experienced by the ions, $\left(\omega_{i}=2 \pi \times 646 \mathrm{kHz}\right)$,

$\omega_{\mathrm{d}} \quad=\gamma^{2} \eta \omega_{e l}^{2} / \omega_{\mathrm{Le}}$ is the electron drift frequency in the space-charge field of the ions $\left(\omega_{d}=2 \pi \times 291 \mathrm{kHz}\right)$.

The values for the frequencies given in parenthesis above are for "LEAR standard conditions" as defined in Table 1 (where for simplicity trapped hydrogen ions are assurned).

The r.h.s. of Eqs. (1.1) and (1.2) describe the external excitation by kicker electrodes (used e.g. for diagnostics). For a short electrostatic kicker of length $\ell$ at $\mathrm{z}=0$ with a horizontal electric field $E e^{-i \omega t}$ one has e.g.:

$$
F_{e}=\frac{e}{m_{e} \gamma} E l \delta(z), \quad F_{i}=\frac{-e Z}{M} E l \delta(z) .
$$


Table 1 : LEAR cooler "standard parameters".

\begin{tabular}{|l|l|l|}
\hline Electron energy & $E_{e}$ & $2.8 \mathrm{keV}$ \\
\hline Velocity factor & $\beta=v_{e} / c$ & 0.1 \\
\hline Electron current & $I_{e}$ & $0.3 \mathrm{~A}$ \\
\hline Electron density & $\mathrm{n}_{\mathrm{e}}$ & $3.2 \times 10^{13} \mathrm{~m}^{-3}$ \\
\hline Longitudinal B-field & $\mathrm{B}$ & $0.063 \mathrm{~T}$ \\
\hline Length of neutral. section & $\mathrm{L}$ & $3.2 \mathrm{~m}$ \\
\hline Neutralisation factor & $\eta$ & 0.4 \\
\hline Ion charge to mass ratio & $\mathrm{Z} / \mathrm{A}$ & 1 \\
\hline
\end{tabular}

For an infinitely long system we seek solutions of Eqs. (1) in the following form:

$$
U_{e, i}=a_{e, i} \exp (i k z-i \omega t), \quad \tilde{U}_{e, i}=\tilde{a}_{e, i} \exp (i k z-i \omega t)
$$

We obtain:

$$
\begin{aligned}
& a_{e}\left[\left(1 / I_{e}\right)+\omega_{e l}^{2}\right]-a_{i}\left(\eta \gamma^{2} \omega_{e l}^{2}\right)=f_{e} \\
& -a_{e}\left(\omega_{i o n}^{2}\right)+a_{i}\left[\left(1 / I_{i}\right)+\eta \omega_{i o n}^{2}\right]=f_{i}
\end{aligned}
$$

Here the dispersion integrals are:

$$
\begin{aligned}
I_{i} & =\int \frac{f\left(v_{i}\right)}{\omega_{i}^{2}-\omega_{L i}\left(k v_{i}-\omega\right)-\left(k v_{i}-\omega\right)^{2}} d v_{i} \\
& =\frac{1}{2 \sqrt{\left(\omega_{L i} / 2\right)^{2}+\omega_{i}^{2}}}\left\{\int \frac{f\left(v_{i}\right)}{\omega_{i 1}-\left(\omega-k v_{i}\right)} d v_{i}-\right. \\
& \left.-\int \frac{f\left(v_{i}\right)}{\omega_{i 2}-\left(\omega-k v_{i}\right)} d v_{i}\right\} \\
I_{e} & =\int \frac{f\left(v_{e}\right)}{\omega_{e}^{2}-\omega_{L e}\left(k v_{e}-\omega\right)-\left(k v_{e}-\omega\right)^{2}} d v_{e} \\
& \approx \int \frac{f\left(v_{e}\right)}{\omega_{e}^{2}+\left(\omega-k v_{e}\right) \omega_{L e}} d v_{e}
\end{aligned}
$$

Note that in the second expression for $I_{i}$

$$
\omega_{i 1,2} \equiv \frac{\omega_{L i}}{2} \pm \sqrt{\left(\frac{\omega_{L i}}{2}\right)^{2}+\omega_{i}^{2}}
$$

is introduced and in the approximation for $I_{e}$ it is assumed that $\omega_{L e}>>\left|\omega_{e}-k v_{e}\right|$

The quantities $f_{e, i}(k)=\frac{1}{L} \int_{0}^{L} F_{e, i} e^{-i k z} d z$ (with $k=n \pi / L$, $n=0,1,2 \ldots$, and $L$ the length of the neutralisation section) in Eq. (2) are the Fourier expansion coefficients of the kicker fields $F_{e, i}(z)$. Only the leading harmonic (close to the resonance to be determined below) is retained. For large $\mathrm{L}$ the spectrum of $\mathbf{k}$ values is practically continuous.

The behaviour of the system (2) is given by the determinant:

$$
D=\left|\begin{array}{cc}
\left.\left(1 / I_{e}\right)+\omega_{e l}^{2}\right] & -\eta \gamma^{2} \omega_{e l}^{2} \\
-\omega_{i o n}^{2} & {\left[\left(1 / I_{i}\right)+\eta \omega_{i o n}^{2}\right.}
\end{array}\right|
$$

The solution of the homogenous equation $\left(F_{e}=F_{i}=0\right)$ is determined by $\mathrm{D}=0$.

With external excitation a solution of the system (2) is

$$
\begin{aligned}
& a_{e}=\frac{f_{e}\left[\left(1 / I_{i}\right)+\eta \omega_{i o n}^{2}\right]+f_{i}\left(\eta \gamma^{2} \omega_{e l}^{2}\right)}{D} e^{-i k z} \\
& a_{i}=\frac{f_{e}\left(\eta \omega_{i o n}^{2}\right)+f_{i}\left[\left(1 / I_{e}\right)+\omega_{e l}^{2}\right]}{D} e^{-i k z}
\end{aligned}
$$

It exhibits resonant behaviour for $D(k, \omega) \rightarrow 0$. For a system of finite length, the situation becomes very complex because boundary conditions have to be satisfied, which can e.g. be accomplished by adding solutions corresponding to reflections at the borders [3]. This will not be discussed any further here. Rather we content ourselves with the solution of the homogenous equation for an unbounded system.

\section{STABILITY CONDITIONS}

We discuss the solution of the homogeneous equation for the case of negligible dispersion of the electrons, $f\left(v_{e}\right)$ $=\delta\left(v_{e}\right)$. Then the characteristic equation $\mathrm{D}=0$ is approximated by:

$$
\left\{\omega_{d}-\left(k \nu_{e}-\omega\right)\right\}\left\{\left(1 / I_{i}\right)+\eta \omega_{\text {ion }}^{2}\right\}-\omega_{\text {ion }}^{2} \omega_{d}=0
$$

For negligible ion velocity spread, the term $\left\{\left(1 / I_{i}\right)+\eta \omega_{i o n}^{2}\right\}$ becomes $\omega_{\text {ion }}^{2}+\omega_{L i} \omega-\omega^{2}$. The roots of this expression define the "coherent ion frequencies"

$$
\omega_{i c o h 1,2}=\left|\frac{\omega_{L i}}{2} \pm \sqrt{\left(\frac{\omega_{L i}}{2}\right)^{2}+\omega_{i o n}^{2}}\right|
$$

(which for the parameters of Table 1 have the values $\omega_{\text {icoh } 1}=2 \pi \times 1.4 \mathrm{MHz}, \quad \omega_{\text {icoh } 2}=2 \pi \times 0.48 \mathrm{MHz}$ ). With finite spread of the ion velocity, developing the denominator of $I_{i}$ into a Taylor series up to second-order in $k v$ and using $\int f\left(v_{i}\right) v d v=0, \int f\left(v_{i}\right) v^{2} d v=\sigma^{2}$. Eq. (6) yields a third-order equation for $k(\omega)$. An estimate of the growing solution can

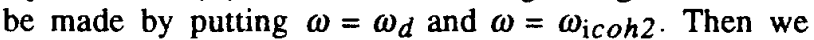
obtain for the imaginary part of $k$

$$
\lambda=\operatorname{Im}(-k)=\frac{\sqrt{3}}{2} \sqrt[3]{\left|\frac{\omega_{\text {ion }}^{2} \omega_{d}}{\nu_{e} \sigma^{2} F}\right|}
$$

with $\mathrm{F}=\left[4+\left(\omega_{L i}^{2} / \omega_{i o n}^{2}\right)-\eta\right] / \eta \approx 4 / \eta$. Thus pertubations can be amplified by a factor of $K=\mathrm{e}^{\lambda L}$ over the length $(L)$ of the neutralisation section. If this is too large $\left[\lambda L \geq \ln \left(K_{C}\right)\right]$ the system is unstable. The limiting amplification $K_{c}$ depends on the initial perturbation and/or on the "internal feedback", e.g. by reflected electrons, to be discussed below. 
The upper limit for the stable neutralisation (substituting $\omega_{\text {ion }}, \omega_{d}$, etc. in Eq. (7)) can be expressed as:

$$
1.27 \times 10^{-9}\left[\frac{\mathrm{m}^{6} \mathrm{~T}}{\mathrm{~s}^{3}}\right] \frac{n_{e}^{2}}{v_{e} B}\left(\frac{Z}{A}\right) \frac{\eta^{2}}{\sigma^{2}} \cdot L^{3} \leq\left|\ln K_{c}\right|^{3}
$$

We conclude that a large spread $\sigma$, a large field $B$ and ions of large $(A / Z)$ ensure a high degree of neutralisation $\eta$.

A crucial parameter is the longitudinal velocity spread $(\sigma)$ of the ions. Heating due to collisions with the electrons yields a relatively low $(\leq 1 \mathrm{eV})$ ion temperature compared to the transverse energy spread (typically $100 \mathrm{eV}$ ) in the Coulomb field of the electron beam. For a long ion lifetime (ultra high vacuum) one can (perhaps) assume that this transverse spread couples into the longitudinal plane. One potential mechanism for this involves the reflections in the field of the trapping electrodes at either end of the neutralisation section. Thus we estimate $\sigma^{2}=c^{2} \frac{k \Delta T_{e}}{M c^{2}}$ where $\mathrm{k} \Delta \mathrm{T}_{\mathrm{e}}[\mathrm{eV}]=(30 / \beta) I_{e}[\mathrm{~A}]$ is the potential difference (centre to edge) in the electron beam.

Reflected electrons escaping from the collector or the migrating ions themselves can feed the perturbation back to the upstream end. Stability of the system requires $\alpha \mathrm{K} \leq 1$ where $\alpha$ is the "feedback coefficient" i.e the fraction of the perturbation taken back to the entrance. For typical conditior s (Table 1 ) and taking an ion temperature $0.5 M \sigma^{2}$ of $50 \mathrm{eV}$, Eq. (7) yields $\lambda \approx 2.1, \lambda \mathrm{L} \approx 6.7, \mathrm{~K} \approx 800$. Thus only a very small "internal feedback" $(\alpha<1 / 800)$ is tolerable. The current loss $\Delta I_{e} / I_{e}$ from the collector can be as high as $2 \times 10^{-3}$ if the beam is badly adjusted, but probably only a fraction of the reflected electrons will get back to the upstream end with the right phase for the growth. Thus we expect $\alpha<\Delta I_{e} / I_{e}$. Nevertheless, a high collector efficiency and/or clearing of reflected electrons is important. This has been confirmed both at the test-bench at the CAPT in Russia and at the LEAR electron cooler. In addition, any other internal feedback has to be small to ensure stable neutralisation.

\section{EXTERNAL FEEDBACK AND "SHAKING"}

To suppress the instability in the regime where the "internal feedback" or the initial perturbations are strong, an external feedback system has been implemented at LEAR. The signal is taken from a pickup, near one end of the cooling section. It is duly amplified and phase shifted before being applied to a kicker at the other end of the cooler.

The preliminary tests are commented in Ref. [4]. So far we have been unable to stabilise a strongly unstable beam by this system. It was however possible to obtain a stable primary e-beam by "shaking" with a pure sine-wave signal applied to a transverse kicker. We observe from the examples given above that all the characteristic ion frequencies are in the 0.1 to $2 \mathrm{MHz}$ region. By exciting at these frequencies we expect strong response. This was confirmed by the experiments. The response showed abrupt discontinuities at frequencies corresponding to the resonances of ions of the different masses which can thus be selectively expelled. In this way we have been able to obtain stable neutralisation at values which could be chosen between zero ("clearing by shaking") and a maximum somewhat lower than the one reached during fluctuations without shaking (Table 2).

Table 2 : An example of the effect of shaking

\begin{tabular}{|l|l|l|}
\hline Electron energy & \multicolumn{2}{|c|}{$2.8 \mathrm{keV}$} \\
\hline Electron current & \multicolumn{2}{|c|}{$0.3 \mathrm{~A}$} \\
\hline Voltage on trapping electrodes & 0 & $6 \mathrm{kV}$ \\
\hline $\begin{array}{l}\text { Neutralisation without shaking } \\
\text { fluctuating between }\end{array}$ & $0-0.2$ & $0.5-0.7$ \\
\hline $\begin{array}{l}\text { Neutralisation with shaking } \\
\text { stable at }\end{array}$ & 0 & 0.4 \\
\hline
\end{tabular}

The response of the beam ion system both with and without external excitation is also a powerful diagnostic tool $[1,4]$. The onset of the instability is accompanied by strong RF signals at the coherent frequencies of one or several ion species. It appears that ions of $(A / Z) \approx 4-6$ remain trapped. The relatively large width of the resonance supports the hypothesis of a large velocity spread which is an essential ingredient of the theory described in the present paper.

\section{CONCLUSIONS}

The theory sketched above explains - at least in a qualitative way - the observations made at LEAR. Several refinements, e.g. the non-linearity of the space-charge fields as well as initial and boundary conditions for the finite system, have to be included before quantitative agreement can be expected. Stable electron beams of $0.3 \mathrm{~A}$ at $2.8 \mathrm{keV}$ have been achieved with a neutralisation of up to $40 \%$. A more detailed analysis of the internal and external feedback is required to see whether further improvements are feasible.

\section{REFERENCES}

[1] A.V. Burov, V.I. Kudelainen, V.A. Lebedev, V.V. Parkhomchuk, A.A. Sery, V.D. Shiltsev, Experimental Investigation of an Electron Beam in Compensated State, CERN/PS 93-03 (AR), 1993 (Translation of Novosibirsk Preprint 89-116).

[2] A.V. Burov, Secondary Particle Instabilities in the Storage Rings with Electron Cooling, Proc. Workshop on Beam Cooling ..., CERN 94-03, 1994, p.230.

[3] M.V. Nezlin, Physics of Intense Beams in Plasmas, Institute of Physics, Bristol 1993.

[4] J. Bosser, F. Caspers, M. Chanel, R. Ley, R. Maccaferri, S. Maury, I. Meshkov, G. Molinari, V. Polyakov, A. Smimov, O. Stepashkin, A. Syresin, G. Tranquille, F. Varenne, Neutralisation of the LEAR Electron Cooling Beam ..., this conference. 
